\title{
The Application of Digital Simulation Technology In Ship Power System
}

\author{
Yuan Renmin \\ Marine college Shandong jiaotong university, China \\ e-mail: yrm0019@163.com
}

Keywords:Object-oriented technology; marine electric power system; building block design; layered structure

\begin{abstract}
This paper described the ship power system digital simulation technology is object-oriented simulation technology, it adopts the modular design and layered structure, among them, the modular design to enhance the simulation agility and scalability, and hierarchical structure is in reducing modeling workload at the same time greatly improve the simulation efficiency. Therefore, this technology in the ship power system digital simulation field has high practical value and broad application prospect.
\end{abstract}

\section{Introduction}

Ship power system, as an independent comprehensive power supply network, and land use of large power supply network the distinction that having essence.Ship power system load and power comparable, general bulk carrier of winch or ballast pump single power can reach more than 100 $\mathrm{Kw}$ to $300 \mathrm{Kw}$, and even single generator power often is $500 \sim 800 \mathrm{Kw}$.With large and ultra large container ship and electric propulsion ship, the emergence of the thruster and main propulsion motor power is close to or even more than the main power station of single generator power, the power system management and operation control and put forward higher request.Especially in electric propulsion ship power system, with high power transformer, thyristor device, frequency converter, permanent magnet synchronous motor, rotating converter technology combination as the core of the ac change technology has been widely used, and therefore bring power harmonic pollution problems, converter and power transmission system and the interaction between, there is lack of effective evaluation methods.In addition, complicated Marine environment determines the ship power system operation condition, this makes the complexity of ship power station management and use of the stability of the facing problems become more severe.

To solve these problems and defects, need to ship power system a lot of test and research.As the ship power system in most cases do not have in the original equipment directly test conditions.Therefore, with good economic and practical digital simulation get the application and popularization.Based on the reference land power system digital simulation technology was proposed based on a kind of object oriented ship power system digital simulation technology.With the aid of SIMULINK simulation software, the prototype power system digital simulation, so can greatly improve the simulation efficiency, enhance the scalability and vivid, have good application prospect.

\section{Object-oriented Ship Power System Digital Simulation}

Object-oriented digital simulation technology is the core of the knowledge, describe reality system object process and methods and analysis, design, implementation of the system process and methods combined.Accordingly, this paper puts forward the object-oriented ship power system digital simulation technology two outstanding characteristic is the hierarchical structure and modular design.

1.1 layered structure

Ship power system and land power system compared with small, but its complexity did not reduce.This article put forward according to the objects belonging to the class layer, accordingly the whole simulation system is divided into four different levels, adopt hierarchical design, hierarchical simulation, layered combination, comprehensive simulation method to the ship power system 
digital simulation.through the simulation object, the classification of the simulation system formed from simple to complicated layered structure. The layered structure, not only reduce the workload of the design and debugging, more important is to enrich the simulation method, thus greatly improving the efficiency of the simulation.

\section{2 modular design}

In the proposed ship power system digital simulation technology, adopted the modular packaging design, different simulation module with only between input and output relationship, which not only greatly improve the agility and scalability, and at the same time also largely simplified the system structure.

\section{The Simulation Case Analysis}

The fifth generation in modern large container ship power system is a typical ship power system, the main electrical wiring is shown as shown in figure 1. This system has four sets of diesel generator, diesel engine using wartsila four stroke medium speed machine, generator using brushless excitation mode of synchronous generator. Ship power station management system USES the Japanese TERESAKI development of GAC - 16 m microcomputer control system. High power load with cooling box with compressor, bow thruster (piezoelectric equipment), etc.

\section{1 simulation modeling}

In front of the analysis has already pointed out that, according to different object, the simulation system from top to bottom can be divided into four levels. Based on the simulation system of the layered structure, we adopt the layered modeling strategy.

In the hierarchical modeling of first is the view of mathematics layer simulation modeling, its purpose is to make various mathematical model transformation for Matlab mathematical module combination form, as shown in figure 2 shows the excitation system block diagram, as shown in

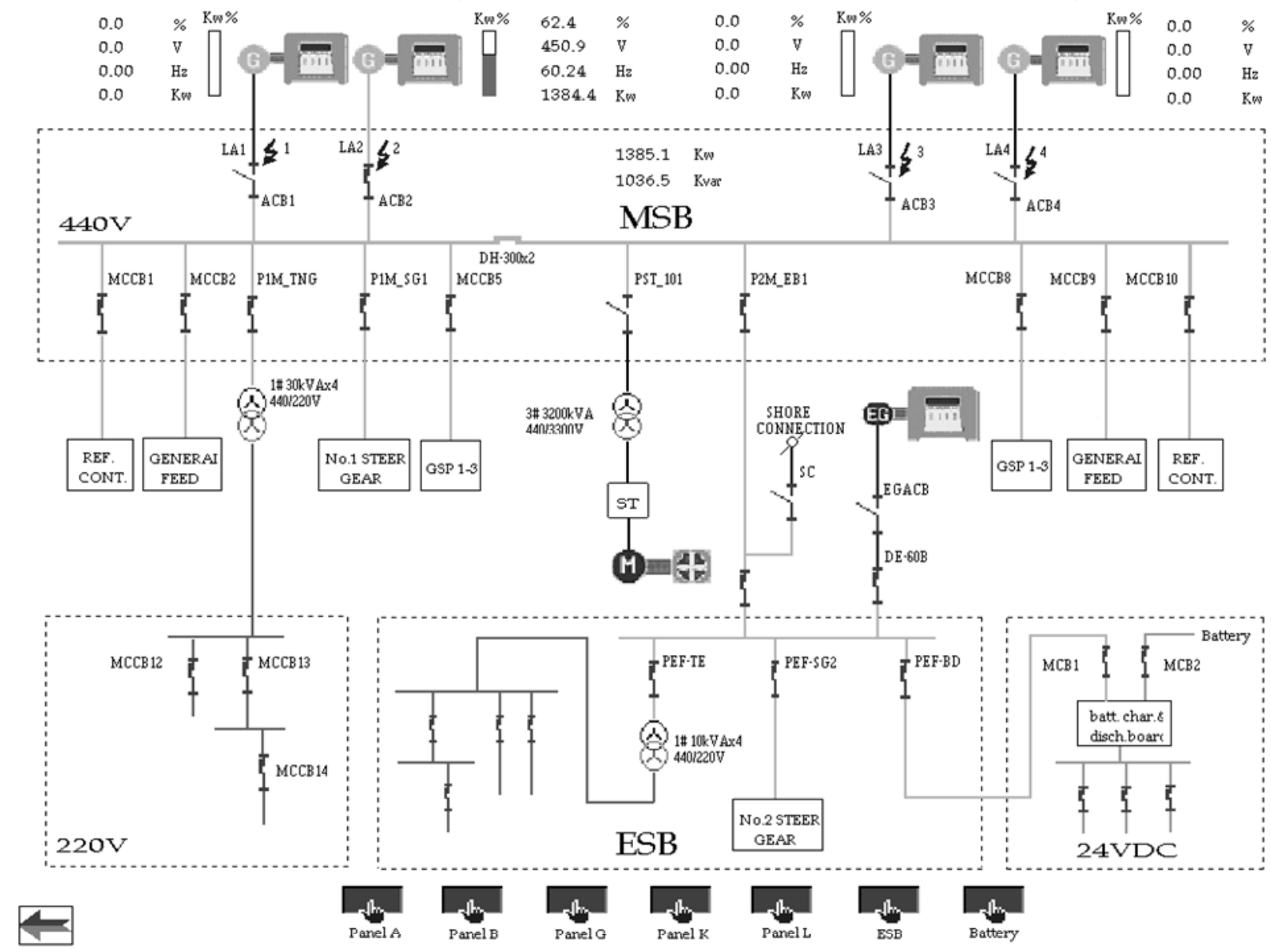

Fig. 1 ship power bus 
figure 3 shows the excitation system transfer function block diagram. In mathematical modeling work layer, on the basis of various model based on the package and combination can form the required component layer of the simulation mode.

Through the above analysis, we can conclude that the hierarchical modeling is from easy to difficult

pattern, and all levels of modeling is relatively independent, so this is largely reduce the modeling workload, but also for the system debugging and maintenance have created favorable conditions.

2. 2 the simulation results analysis

The generator of simulink simulation have shown in figure 4 for a generator starting, and car, process and 92\% working curve. By the above curve is calculated as follows:

Generator starting, and car, process and $92 \%$ load in the transient voltage rate for:

$$
\Delta U_{d} \%=\frac{U_{d}-U_{n}}{U_{n}} \times 100 \%=\frac{396-450}{450} \times 100 \%=-12 \%
$$

Generator starting, and car, process and $92 \%$ load of steady state voltage rate for:

$$
\Delta U_{s} \%=\frac{U-U_{n}}{U_{n}} \times 100 \%=\frac{449.9-450}{450} \times 100 \%=-0.023 \%
$$

After calculation, generator voltage change rate and recovery time all can meet steel ships classification norms, and prototype use consistent.

\section{Summary}

In this paper, the object-oriented ship power system digital simulation technology has layered structure and the characteristics of modular design. The application of layered structure can reduce the simulation modeling workload and improve the simulation efficiency, and modular design is to enhance the simulation system agility and scalability. Through to the fifth generation container ship power system digital simulation results show that the proposed ship power system digital simulation modeling method is correct and feasible, it for ship power system research provides an effective tool.

\section{References}

[1] Hodge C G, Mattick D J, Flower J O. Novel converters for electric ship propulsion system and shipboard power distribution[A]. IEEE Conference Record of Power Modulator Symposium[C].2000: 89 96.

[2]Calfo R M, Fulmer J A, Tessaro J E. Generators for use in electric marine ship propulsion systems[A]. Power Engineering Society Summer Meeting, 2002 IEEE[C]. Vol. 1, 254 259.

[3]Sun Caiqin,Guo Chen,Shi Chengjun. Modeling and simulating to large intelligent marine power station system. Proceedings of the World Congress on Intelligent Control and Automation (WCICA),2006:6128-6132.

[4]Adnanes, A.K. (2003), Maritime Electrical Installations and Diesel Electric Propulsion, Tutorial Report/Textbook, ABB Marine AS, Oslo, Norway, 2003

[5]Xu Shang ling. “Marine Engine Automation”. Dalian maritime affairs university press. China. 2001: p.291-299.

[6]Liu Xingqiao, Shi Zhenwei, Yao Yanchun. Control system of loading manipulator. Proceedings of the Eighth International Conference on Electrical Machines Systems, 2005:1507-1512. 


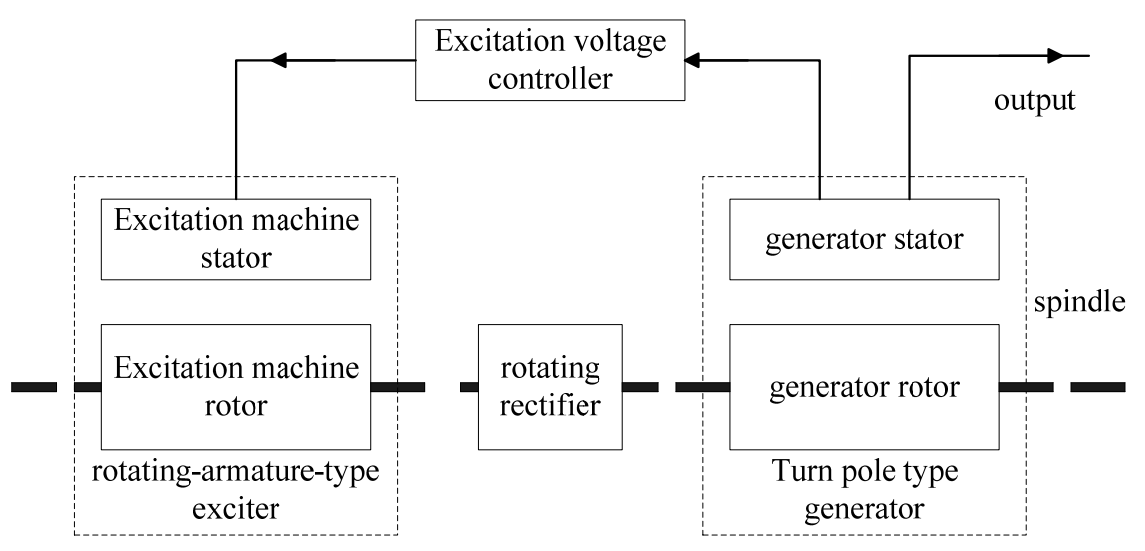

Fig. 2 Structure of Alternate Current Excitation

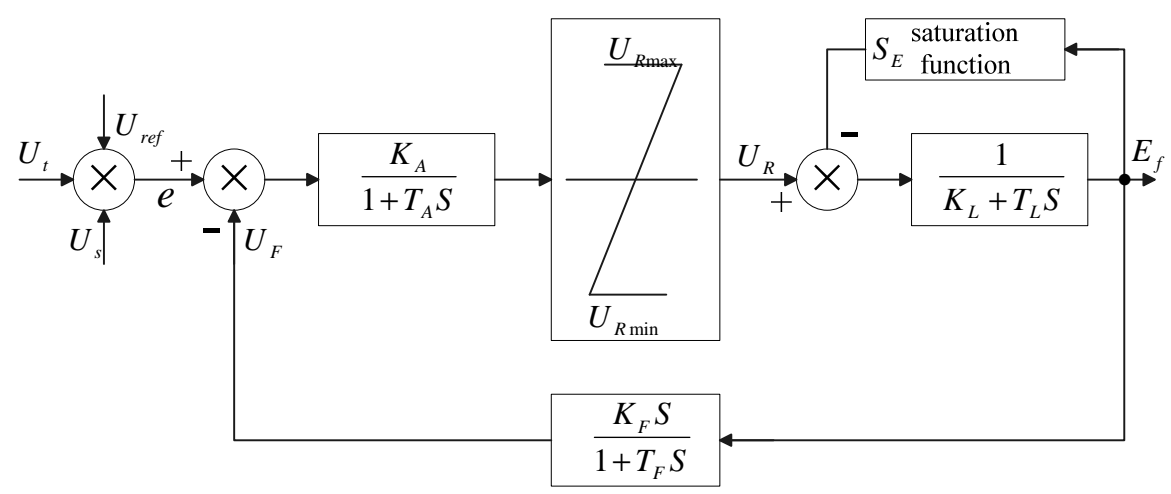

Fig. 3 Transfer Function of Excitation System

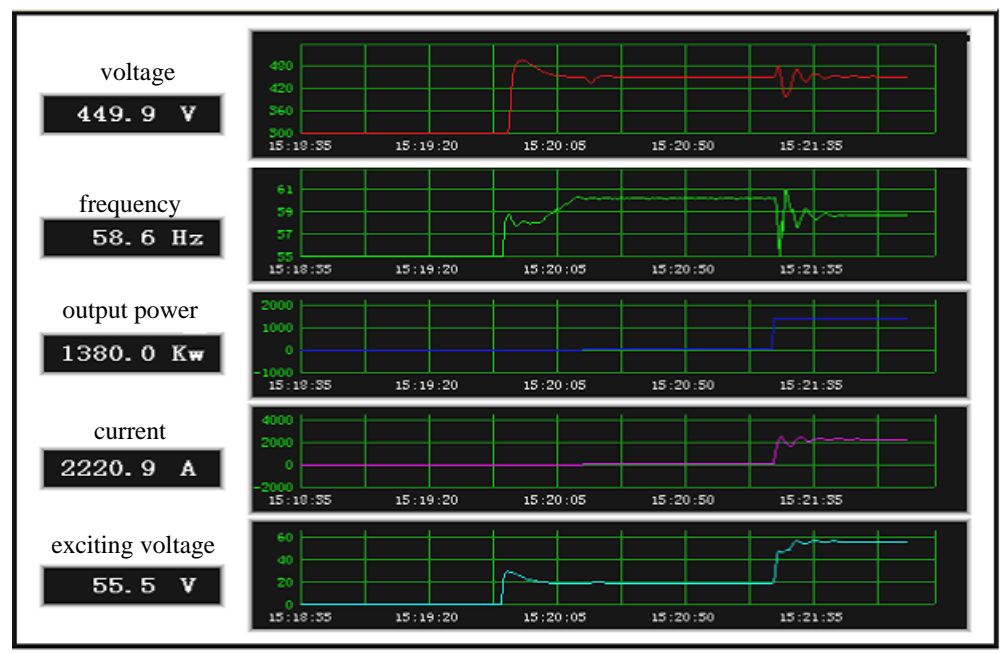

Fig. 4 Generator parameter curve 